OPEN ACCESS

Edited by:

Johannes Wolfgang Dietrich, Ruhr University Bochum, Germany

Reviewed by: Isabel Moreno Indias, Universidad de Málaga, Spain Yaron Tomer, Albert Einstein College of Medicine, United States

*Correspondence: Zhongyan Shan shanzhongyan@medmail.com.cn

Specialty section: This article was submitted to Thyroid Endocrinology, a section of the journal Frontiers in Endocrinology

Received: 11 September 2021 Accepted: 29 October 2021 Published: 17 November 2021

Citation:

Gong B, Wang C, Meng F, Wang $H$, Song B, Yang Y and Shan Z (2021) Association Between Gut Microbiota and Autoimmune Thyroid Disease: A Systematic Review and Meta-Analysis.

Front. Endocrinol. 12:774362. doi: 10.3389/fendo.2021.774362

\section{Association Between Gut Microbiota and Autoimmune Thyroid Disease: A Systematic Review and Meta-Analysis}

\author{
Boshen Gong, Chuyuan Wang, Fanrui Meng, Haoyu Wang, Bo Song, \\ Yang Yang and Zhongyan Shan *
}

Department of Endocrinology and Metabolism, Institute of Endocrinology, National Health Commission (NHC) Key Laboratory of Diagnosis and Treatment of Thyroid Diseases, The First Affiliated Hospital of China Medical University, China Medical University, Shenyang, China

Background: Autoimmune thyroid disease (AITD) is characterized by thyroid dysfunction and deficits in the autoimmune system. Growing attention has been paid toward the field of gut microbiota over the last few decades. Several recent studies have found that gut microbiota composition in patients with AITD has altered, but no studies have conducted systematic reviews on the association between gut microbiota and ATID.

Methods: We searched PubMed, Web of Science, Embase, and Cochrane databases without language restrictions and conducted a systematic review and meta-analysis of eight studies, including 196 patients with AITD.

Results: The meta-analysis showed that the alpha diversity and abundance of certain gut microbiota were changed in patients with AITD compared to the controls. Chao1,the index of the microflora richness, was increased in the Hashimoto's thyroiditis group compared to controls (SMD, $0.68,95 \% \mathrm{Cl}: 0.16$ to 1.20$)$, while it was decreased in the Graves' disease group (SMD, $-0.87,95 \% \mathrm{Cl}:-1.46$ to -0.28$)$. In addition, we found that some beneficial bacteria like Bifidobacterium and Lactobacillus were decreased in the AITD group, and harmful microbiota like Bacteroides fragilis was significantly increased compared with the controls. Furthermore, the percentage of relevant abundance of other commensal bacteria such as Bacteroidetes, Bacteroides, and Lachnospiraceae was increased compared with the controls.

Conclusions: This meta-analysis indicates an association between AITD and alteration of microbiota composition at the family, genus, and species levels.

Systematic Review Registration: PROSPERO, identifier CRD42021251557.

Keywords: autoimmune thyroid disease, gut microbiota, Hashimoto thyroiditis (HT), Graves' disease (GD), meta-analysis 


\section{INTRODUCTION}

Hashimoto's thyroiditis (HT) and Graves' disease (GD) are the main types of autoimmune thyroid disease (AITD). AITDs are the most common organ-specific autoimmune disorders. Although the clinical manifestations of GD and HT are different, such as hyperthyroidism and hypothyroidism, respectively, GD and HT share similar immune-mediated mechanisms of disease, even alternating from one to the other $(1,2)$. Many studies have revealed the possible causes of AITD, such as genetic susceptibility factors, dysregulation of the immune system, inflammation, stress, and other environmental factors; however, its etiology remains unclear $(3,4)$.

Emerging evidence suggests that the alterations of the gut microbiota play a key role in the development and progress of AITD in individuals. From the embryology aspect, the thyroid and gut share a common embryological origin, explaining some morphological and functional similarities between the gut and thyroid follicular cells (5). The association between autoimmune thyroid disorders and gut autoimmune disease atrophic gastritis was first described in the early 1960s (6). More recently, owing to the development of the $16 \mathrm{~S}$ ribosomal RNA (16SrRNA) gene sequencing technique, the gut microbiota, which comprises trillions of microorganisms, has been proposed to be involved in the pathogenesis of many autoimmune diseases, such as type 1 diabetes, lupus nephritis, Rheumatoid arthritis, Celiac Disease, and AITD (7-10). Although there is no direct evidence that AITD and gut microbiota have a cause-effect relationship, several studies have suggested that the thyroid-gut axis has beneficial or detrimental effects on thyroid function (11). The gut microbiota shapes the thyroid mainly through the following possible microbial-related mechanisms. First, dysbiosis leads to the damaged intestinal barrier and increased intestinal permeability, allowing the antigens to pass into the circulation and activate the immune system (12). Second, the antibodies in the circulation may react with the bacterial antigen and enhance the activation of the inflammasome in the thyroid gland (13). Guo et al. (14) has found that the expression of the inflammasome, including the NOD-like receptor (NLR) family pyrin domain containing 3 (NLRP3), AIM2, caspase-1, and IL$1 \beta$ mRNA and protein from patients with HT, was significantly increased, which can be regulated by the gut microbiota and its metabolism (15-17). Third, the short-chain fatty acids (SCFAs), metabolites of commensal bacteria fermentation of dietary fiber, are speculated to play a crucial role in the development, functioning, and modulation of the immune system $(18,19)$. For example, butyrate, a SCFAs, is associated with reduced levels of TNF- $\alpha$, IL- 6 and suppressed activation of the NLRP3 inflammasome via GPR109A (20).

Recently, many researchers have found that AITD patients have reduced $\alpha$ diversity and abundances of certain microbiota compared with healthy controls (21-23). The $\alpha$ diversity mainly contains community diversity (Simpson and Shannon) and community richness indices (ACE and Chaol) (24). Among the AITD patients, those with HT tend to have a higher Chaol value than healthy volunteers; however, patients with GD have a lower Chaol value than the controls $(25,26)$. In addition, the current results revealed the correlation between the clinical parameters of AITD, such as TRAb or TPOAb and the certain microbiota $(22,27,28)$. For example, Chen et al. (29) found that the proportion of Synergistetes was negatively correlated with TRAb, and Jiang et al. (30) found that Lactobacillus was positively correlated with TRAb. At the phylum level, Yang et al. (31) found a higher Firmicutes/Bacteroidetes ratio in GD patients than in the control group, which may be relevant to inflammation disease, whereas Hanaa et al. (32) found that the ratio was significantly decreased in patients with AITD. Due to different conflicting data, a further study of the association between the gut microbiota and AITD is needed. To better understand the potential role of gut microbiota in the pathogenesis of AITD,we carried out a meta-analysis to assess the alteration in the microbial population between patients with AITD and healthy controls at different levels.

\section{MATERIALS AND METHODS}

\section{Search Strategy}

We conducted a systematic literature search in PubMed, Web of Science, Embase, and Cochrane databases up to August 2021 using the following search string: (thyroiditis OR Hashimoto Disease OR Thyroiditis, Autoimmune OR Hashimoto Thyroiditis OR Thyroiditis, and Chronic Lymphocytic OR Chronic Lymphocytic Thyroiditis OR Thyroid Diseases OR Graves' Disease OR Disease, Graves OR Goiter, Exophthalmic OR Basedow's Disease OR Hyperthyroidism, Autoimmune) AND (microbiota OR Gut Microbiome OR Microbial Community OR Microbial Community OR Gastrointestinal Microbiome OR Microbiome OR Gut Flora OR Gastrointestinal Microbiota OR Microflora, Gastrointestinal OR Gastric Microbiome OR Intestinal Microbiota OR Intestinal Flora).

\section{Inclusion and Exclusion Criteria}

Studies were considered eligible if they met the following criteria: 1) investigating the gut microbiota and patients diagnosed with AITD. 2) providing sufficient data on the relationship between AITD and intestinal microbiota and could be extracted to analyze the 95\% confidence interval (CI). 3) written in English. 4) full-text availability. However, comments, animal model subjects, conference abstract, and reviews were excluded. We also excluded studies with incomplete outcome data on the percentages or relative abundance of gut microbiota and studies with fewer than 20 participants.

\section{Data Extraction}

Three reviewers independently extracted the following data from each study: authors, publication year, country of population, population age, clinical parameters of thyroid function, diagnosis of AITD, and microbiology assessment methods. 


\section{Quality Assessment}

Two reviewers completed the quality assessment using the Newcastle-Ottawa scale (NOS) to evaluate all the included studies, comprising the trial group selection, comparability, and exposure. The total score ranged from 5-9, where a higher score represents a higher quality of assessment. All the discrepancies or poor agreement were resolved through a consensus discussion with a third author.

\section{Statistical Analysis}

Standardized mean differences (SMD) were calculated between the HT and GD groups to assess the bacterial alpha diversity indices (Chao1). SMD $>0$ indicates that participants with HT have a higher level of richness in the intestinal microflora. A fixed- and random-effects were used to assess the percentages or relative abundances of certain gut microbiota with AITD compared with the healthy controls. We examined the statistical heterogeneity using the I2 statistic. I2 values of $25 \%$, $50 \%$, and $75 \%$ were related to low, moderate, and high heterogeneity, respectively. A Random-effects model was used to pool the results when high heterogeneity (I2>50\%) existed. Additionally, the fixed-effects model was used if the heterogeneity was low. Furthermore, we performed a sensitivity analysis as well as Begg's test to assess the potential influences of bias. All statistical analyses were performed using Stata software (version 12.0).

\section{RESULTS}

\section{Characteristics of Included Studies}

The initial literature searches retrieved 282 records from the online database. Among them,92 studies were excluded for duplication, and after the review of titles and abstracts, 175 articles were eliminated because they did not fulfill the inclusion criteria. Then, we evaluated the remaining 15 articles individually; seven were excluded because the studies did not provide quantitative or appropriate data on the gut microbiota relative abundance. Finally, eight studies were included in our meta-analysis (Figure 1). This meta-analysis included 196 patients with AITD and 160 age-matched healthy controls (Table 1). The clinical parameters of AITD and microbiology assessment methods used in the included studies are shown in Table 2. Fecal samples were collected, and microbiota was analyzed by pyrosequencing or high-throughput sequencing of the 16SrRNA gene, real-time PCR, and PCR-DGGE of the 16SrRNA gene (33). The percentage of gut microbiota composition at a different level and relevant abundance were
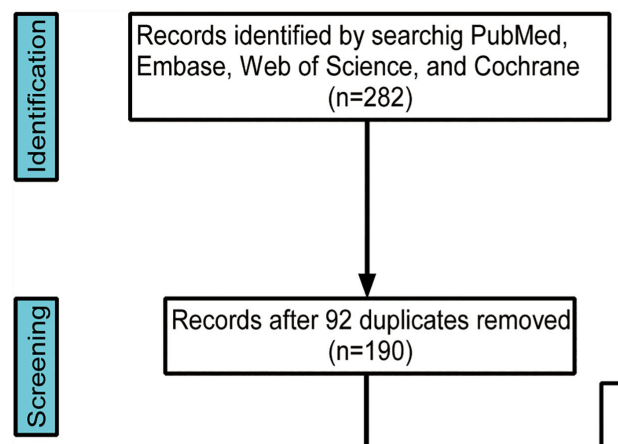

the topic

Animal trial

3. Conference abstract or review

4. In vitro studies
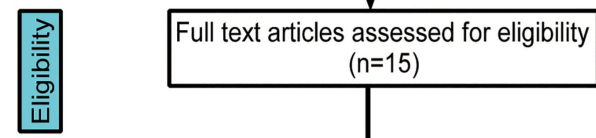

$(n=15)$
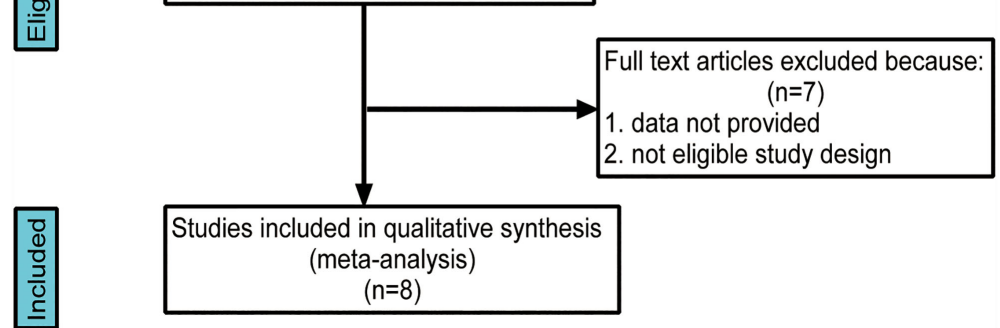

FIGURE 1 | Flow chart of the search strategy and study selection progress. 
TABLE 1 | Characteristics of the studies included in the systematic review and meta-analysis.

\begin{tabular}{|c|c|c|c|c|c|c|}
\hline Author & Country & AITD(n) & Age & Control(n) & Age(years) & Score \\
\hline Zhao (21) & China & 28 & $44.29 \pm 10.7$ & 16 & $44.63 \pm 10.33$ & 8 \\
\hline Zawawy (32) & Egypt & 20 & $38.6 \pm 11.1$ & 30 & $39.7 \pm 10.9$ & 6 \\
\hline Ishaq (25) & China & 29 & $40-60$ & 12 & $40-60$ & 7 \\
\hline Cornejo (28) & Spain & 18 & $40.3 \pm 9.6$ & 11 & $48.8 \pm 6.2$ & 6 \\
\hline Ishaq (26) & China & 27 & $35-50$ & 11 & $35-50$ & 8 \\
\hline Jiang (30) & China & 45 & $16-65$ & 59 & $22-71$ & 6 \\
\hline Chen (29) & China & 15 & $28.87 \pm 6.79$ & 14 & $27.29 \pm 5.73$ & 7 \\
\hline Zhou (33) & China & 14 & $45-65$ & 7 & $48-60$ & 5 \\
\hline
\end{tabular}

AITD, autoimmune thyroid disease.

TABLE 2 | Clinical parameters and microbiology assessment of selected studies.

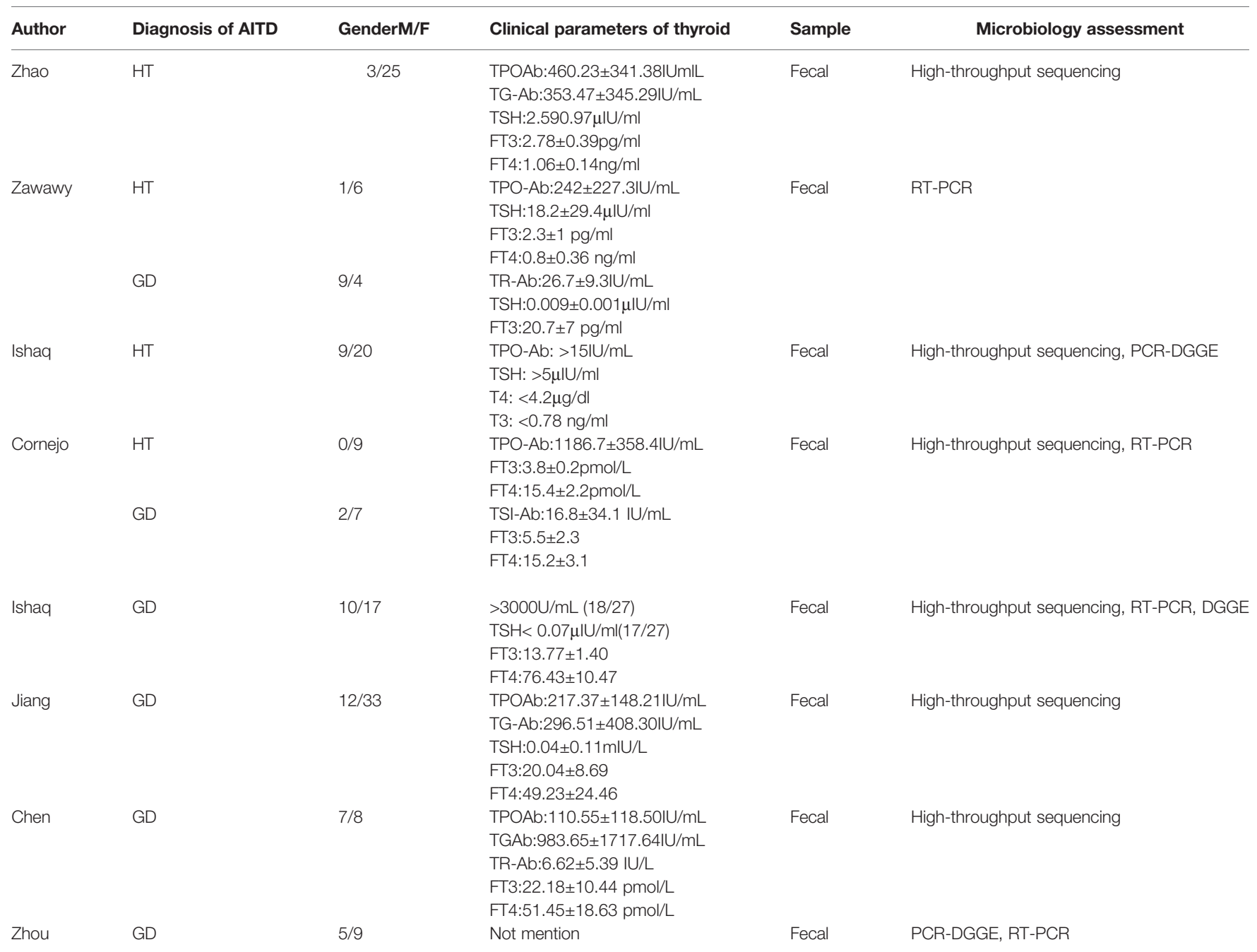

RT-PCR, real time PCR; PCR-DGGE, PCR amplification for Denaturing Gradient Gel Electrophoresis; TPO-Ab, thyroid peroxidase antibody; TG-Ab, thyroglobulin antibodies; TR-Ab, thyrotropin receptor antibodies; HT, Hashimoto Thyroiditis; GD, Graves' disease FT3, Free triiodothyronine; FT4, Free thyroxine; T3, Triiodothyronine; T4, Thyroxine.

analyzed based on the assessment methods, preventing the potential deviation caused by the detection methods.

\section{Alpha Diversity}

The alpha diversity was used to analyze the complexity of species diversity, including diversity indices (Simpson and Shannon) and richness indices (ACE and Chao1). The Chaol index was a reflex of microflora richness. The meta-analysis of four studies reported data about the Chaol index between patients with AITD and healthy controls. We found that the Chaol index was significantly different between the HT and GD groups compared with the controls. To explore the source of heterogeneity, we divided these four articles into two subgroups based on the entities of AITD. The random-effects 
model of subgroup meta-analysis showed that the Chaol index was increased in the HT group compared to the controls (SMD, $0.68,95 \% \mathrm{CI}$ : 0.16 to 1.20 ), while it was decreased in the GD group (SMD -0.87, 95\%CI: -1.46 to -0.28$)$. The effect size $(z=0.14$, $\mathrm{P}=0.886)$ was relatively small. There was no evidence of betweenstudy heterogeneity (I2 = 0\%; Figure 2).

\section{Phylum}

\section{Firmicutes}

Firmicutes, one of the predominant phyla of the human gut microbiota, showed a lower abundance in patients with AITD than the controls (34). A random-effects model showed that the percentage of Firmicutes in the detected sample of HT was $48.3 \%$ (95\%CI: 0.080 to 0.885 ) and $55.1 \%$ (95\%CI: 0.371 to 0.731 ; Figure 3A) in the controls. The effect size $(\mathrm{Z}=6.45, \mathrm{P}=0.000)$ was significant. While it was $41.9 \%$ (95\%CI: 0.163 to 0675 ) in the GD group and $56.6 \%$ (95\%CI: 0.367 to 0.765 ; Figure 3B) in the controls. The effect size $(\mathrm{Z}=5.55, \mathrm{P}=0.000)$ was large and significant. The ratio of bacterial percentage between the HT group (48.3\%) and the control group (55.1\%) was 0.88 (Figure 6A), and that of the GD (41.9\%) group and the controls (56.5\%) was 0.74 (Figure 6B).

\section{Bacteroidetes}

Six studies were used to evaluate the percentage of Bacteroidetes. A random-effects meta-analysis model showed that the percentage of
Bacteroidetes of the HT group was $41.4 \%$ (95\%CI: $0.030-0.798$ ), slightly higher than that in the control group 35.2\% (95\%CI: 0.165 to 0.539 ; Figure $3 \mathrm{C})$. Its effect size $(\mathrm{Z}=5.78, \mathrm{P}=0.000)$ was significant. The same meta-analysis model was used in the GD group; the percentage of Bacteroidetes of the GD group was $52.1 \%$ (95\%CI: 0.227 to 0.815$)$, while the control group was $34.1 \%(95 \%$ CI: 0.141 to 0.540 ; Figure 3D). The effect size $(\mathrm{Z}=5.29, \mathrm{P}=0.000)$ was significant and large. The ratio of bacterial percentage between the HT group (41.4\%) and the control group (35.2\%) was 1.13 (Figure 6A), and between the GD group (52.1\%) and the control group (34.1\%), it was 1.53 (Figure 6B).

\section{Genus}

\section{Bifidobacterium and Lactobacillus}

Probiotics belonging to Bifidobacterium and lactobacillus have been exploited and invented for their benefits in treating many pathological conditions (35). The effectiveness of these two main probiotic strains is used for treatment or therapeutic purposes. A fixed-effects meta-analysis indicated a lower relative abundance of Bifidobacterium in patients with AITD than the controls (SMD -1.08,95\%CI: -1.54 to -0.63 ; Figure 4A). The effect size $(\mathrm{Z}=4.66, \mathrm{P}=0.000)$ was significant. Besides, a fixed-effects model also showed that the relative abundance of lactobacillus decreased compared to that in healthy controls (SMD $-0.86,95 \% \mathrm{CI}:-1.31$ to -0.41 ; Figure $4 \mathrm{~B})$. Its effect size $(\mathrm{Z}=3.78$, $\mathrm{P}=0.000)$ was significant and moderate.

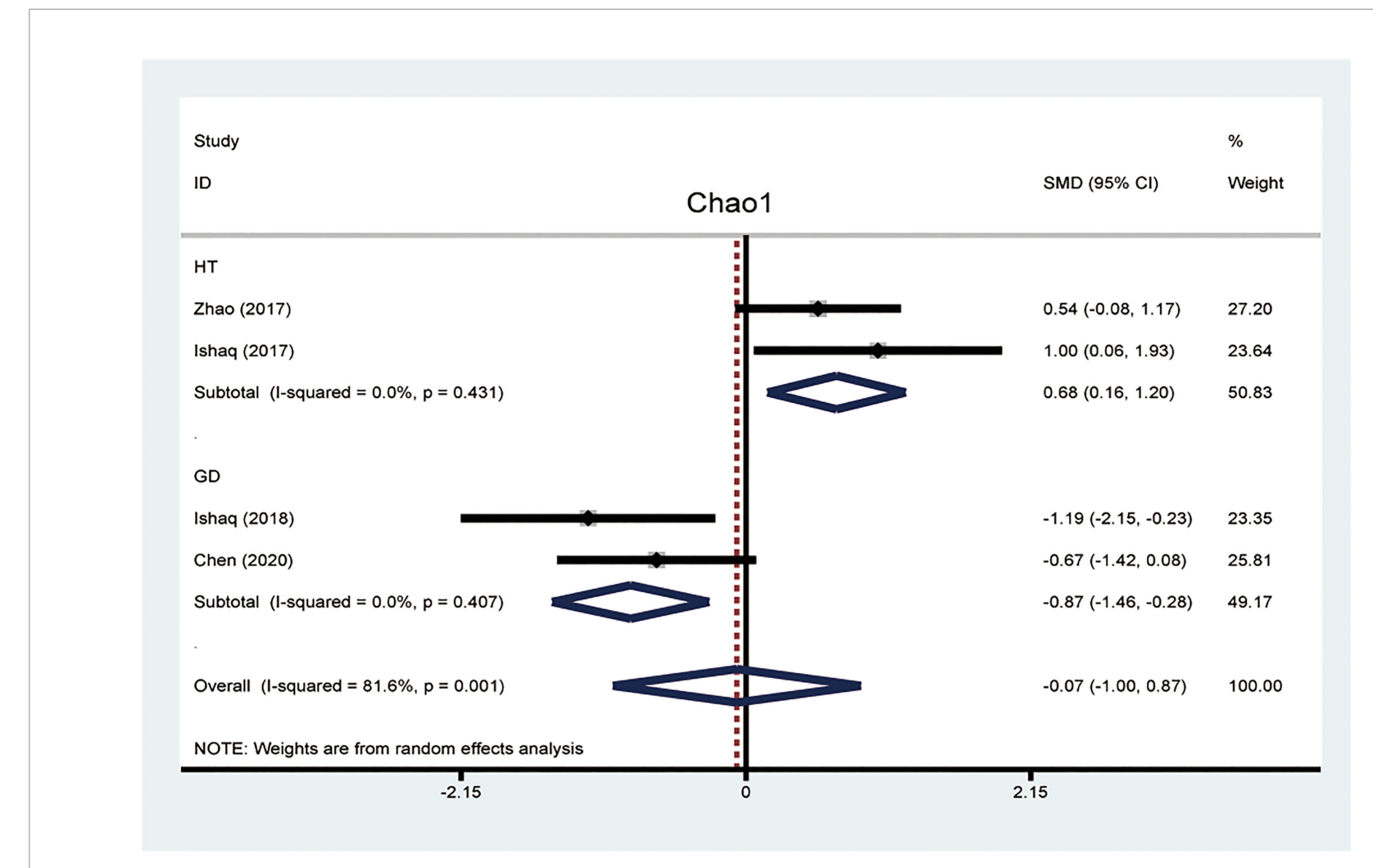

FIGURE 2 | Forest plots of differences of Chao1 index between patients with Hashimoto thyroiditis (HT) and Graves' disease (GD). 
A

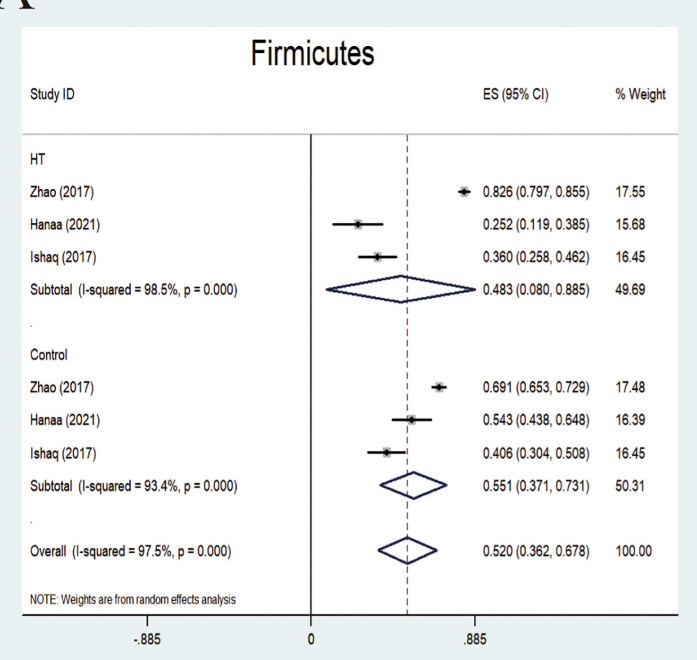

C

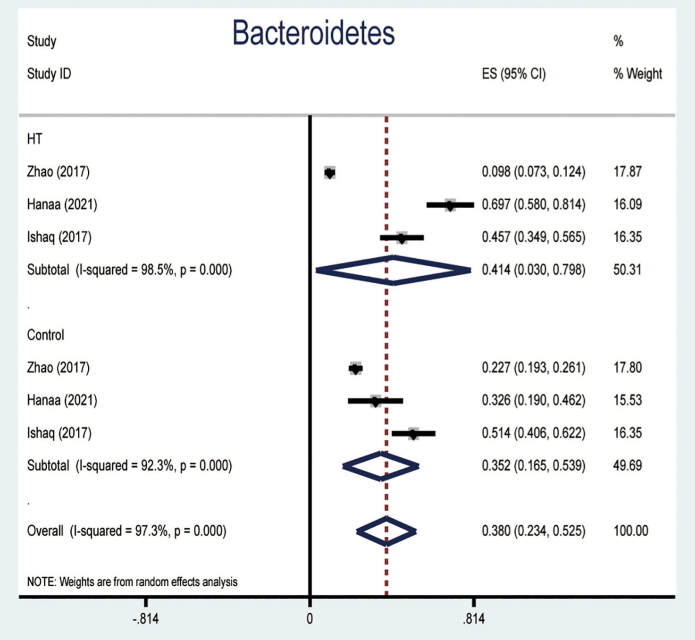

B

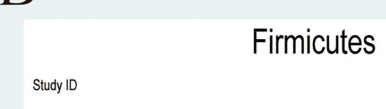

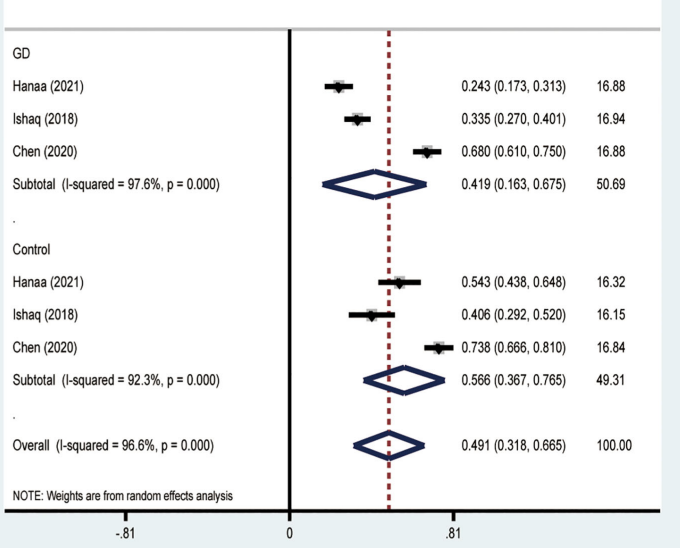
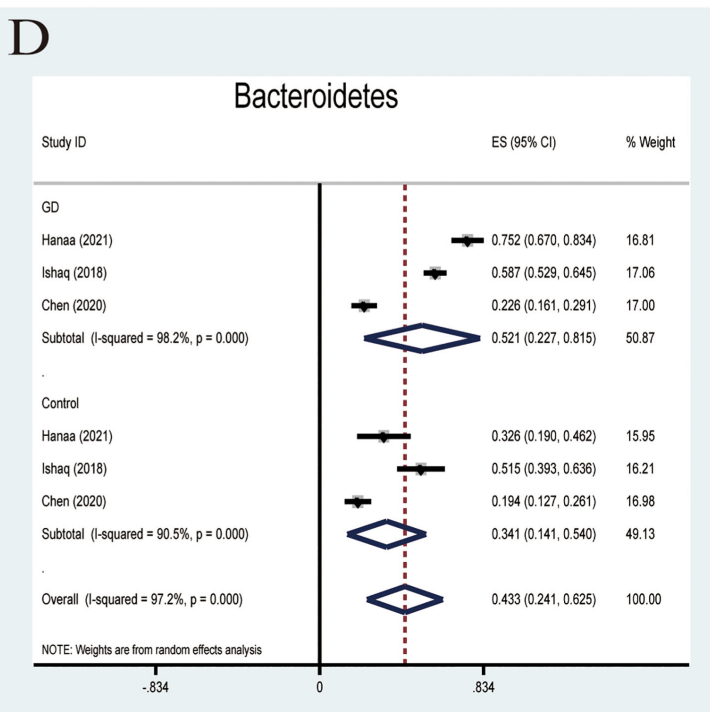

FIGURE 3 | Forest plot of the percentages of Firmicutes and Bacteroidetes comparing people with Graves' disease (GD) or Hashimoto Thyroiditis (HT) to healthy controls. (A-D) Random-effects models were used to assess the percentages of Firmicutes and Bacteroidetes in the detected intestinal microbiota, contributing to higher between-study heterogeneity (I2>50\%).

\section{Bacteroides}

Bacteroides is a pro-inflammatory bacterium that contributes to the pathogenesis of inflammatory bowel disease (36). Five studies analyzed the percentage of Bacteroides in patients with AITD. A random-effects meta-analysis showed $16.4 \%$ of Bacteroides in the detected sample of patients with HT (95\%CI: 0.056 to 0.272 ), while the percentage in healthy control was $14.5 \%$ (95\%CI: 0.110 to 0.181 ; Figure $4 \mathrm{C})$. The effect size $(\mathrm{Z}=5.78, \mathrm{P}=0.000)$ was significant. The percentage of Bacteroides in the GD group was $24.6 \%$ (95\%CI: 0.163 to 0.675$)$ and $18 \%$ (95\%CI: 0.118 to 0.241 ; Figure 4D) in the healthy control group. Its effect size ( $Z=5.29$, $\mathrm{p}=0.000$ ) was significant and large. The ratio of bacterial percentage between the HT group (16.4\%) and the control group (14.5\%) was 1.13 (Figure 6A). In contrast,it was 1.37 between the GD group (24.6\%) and the control group (18.0\%) (Figure 6B).

\section{Family and Species Lachnospiraceae}

Lachnospiraceae, a member of the core gut microbiota, colonizes the host's intestinal tract from birth. Besides, members of Lachnospiraceae are the main microbiota producing SCFA, regulating the inflammasome activation (37). A random-effects meta-analysis showed that the percentage of Lachnospiraceae in the detected sample was $24.3 \%$ (95\%CI: -0.026 to 0.512 ), whereas that in healthy controls was $17.8 \%$ (95\%CI: 0.040 to 

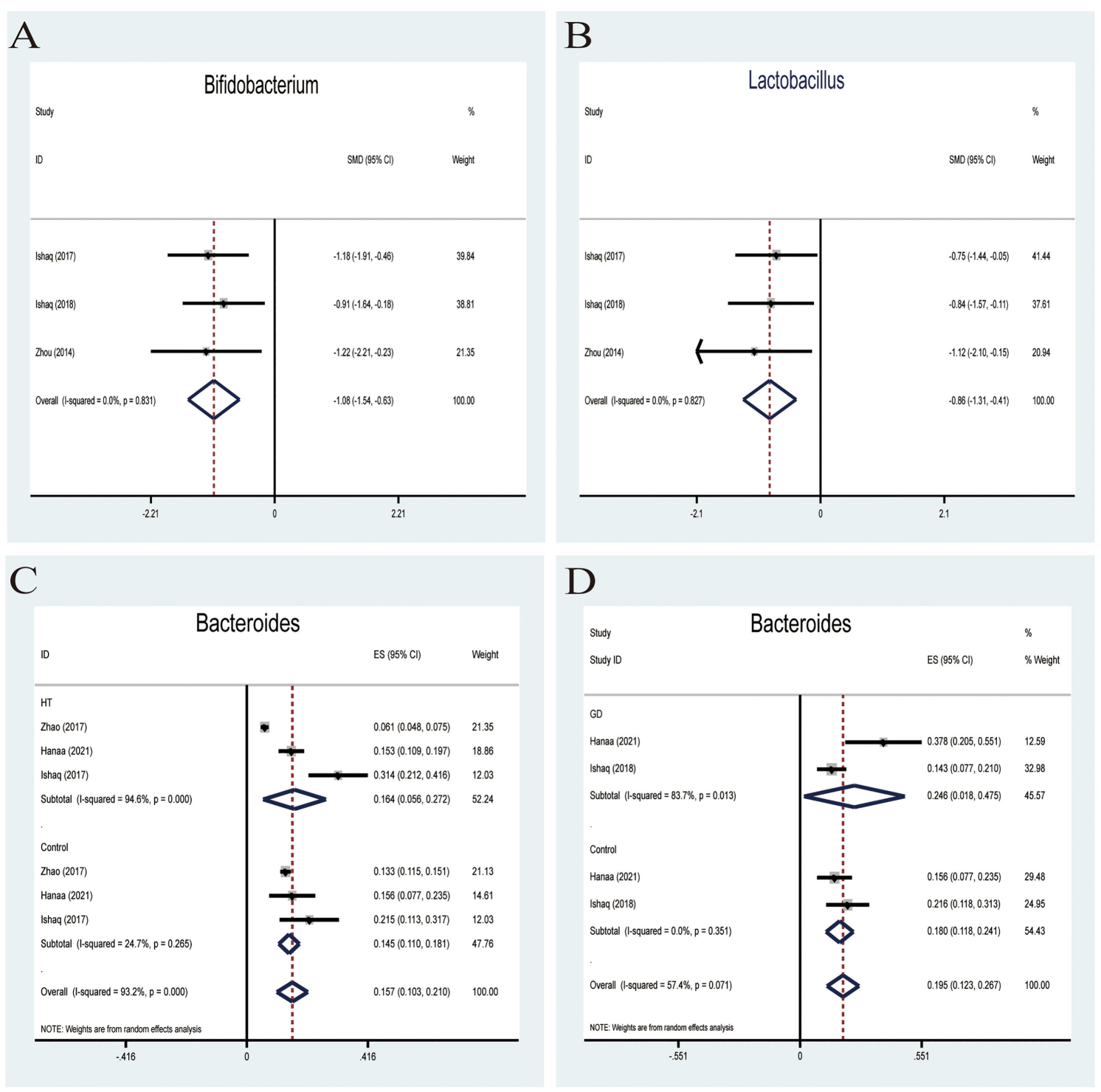

FIGURE 4 | Forest plot of the relative abundance of Bifidobacterium and Lactobacillus and percentages of Bacteroides in autoimmune thyroid disease (AITD). (A, B) Fixed-effects models were used to analyze the relative abundance of Bifidobacterium and Lactobacillus, comparing patients with AITD to healthy controls. (C, D) Random-effects models were used to assess the percentages of Bacteroides, contributing to higher between-study heterogeneity (I2>50\%).

0.316; Figure 5A). The effect size $(\mathrm{Z}=2.89, \mathrm{P}=0.004)$ was significant. The ratio of bacterial percentage between HT (30.3\%) and the control group (21.6\%) was 1.40 (Figure 6A), while between GD $(12.0 \%)$ and the control group (10.3\%), it was 1.17 (Figure 6B).

\section{Bacteroides fragilis}

Bacteroides fragilis was previously reported to stimulate the host immune system by upregulating NLRP3, IL- $1 \beta$,IL-18, and caspase-1 mRNA and protein expression in EGCs and reducing the NLRP6 mRNA expression (38). All four studies reported that $B$. fragilis in the AITD group was significantly higher than that in the controls. A random-effects meta-analysis showed that the percentage of $B$. fragilis in the detected fecal sample was $0.6 \%$ (95\%CI: $0.002-0.011)$ in patients with AITD and $0.1 \%(95 \% \mathrm{CI}: 0.001-0.002)$ in healthy controls. The heterogeneity within the AITD group was low $(29.0 \%$; Figure 5B). Its effect size $(\mathrm{Z}=3.34, \mathrm{P}=0.001)$ was significant. Additionally, the fixed-effects meta-analysis showed that the percentage of Bacteroides fragilis of patients with HT was $0.6 \%$ ( $95 \%$ CI: 0.001 to 0.012 ) compared to $0.4 \%$ in control group ( $95 \%$ CI: 0.000 to 0.007 ; Figure $5 \mathrm{C})$. The effect size $(\mathrm{Z}=2.15, \mathrm{P}=0.031)$ was significant. While the percentage of Bacteroides fragilis of patients with GD was $0.6 \%$ (95\%CI: 0.000 to 0.013 ),higher than the healthy controls $(0.1 \%$; $95 \% \mathrm{CI}: 0.000$ to 0.002 ; Figure 5D). Its effect size $(\mathrm{Z}=2.85, \mathrm{P}=0.004)$ was significant. 


\section{Correlation With Clinical Parameters}

Several studies have revealed the significant correlations between clinical thyroid-related antibodies like TPO-Ab and TR-Ab, and different bacterial abundances by Spearman's correlation distance. The Spearman correlation coefficient R-value between microbiota and immune indices, including TPO-Ab and TR-Ab, demonstrated that certain microbiota significantly correlated with thyroid function tests (Table 3). Among all, the relative abundance of Bacteroides $(\mathrm{R}=-0.342, \mathrm{P}=0.014)$, Dorea $(\mathrm{R}=-$ $0.341, \mathrm{P}=0.022)$, Faecalibacterium $(\mathrm{R}=-0.453, \mathrm{P}=0.014)$, Synergistetes $(\mathrm{R}=-0.711, \mathrm{P}=0.001)$, and Coprococcus $(\mathrm{R}=$ $-0.499, \mathrm{P}=0.03)$ were negatively correlated with TPO-Ab or TR-Ab. In contrast, other commercial bacteria like Blautia, Lactobacillus, Alistipes, Ruminococcaceae, and Enterobacteriaceae were positively correlated with TPO-Ab.

\section{DISCUSSION}

To the best of our knowledge, this is the first meta-analysis to demonstrate the association between gut microbiota and AITD. This association has become a very popular topic and field in recent years. Many researchers want to illuminate the association

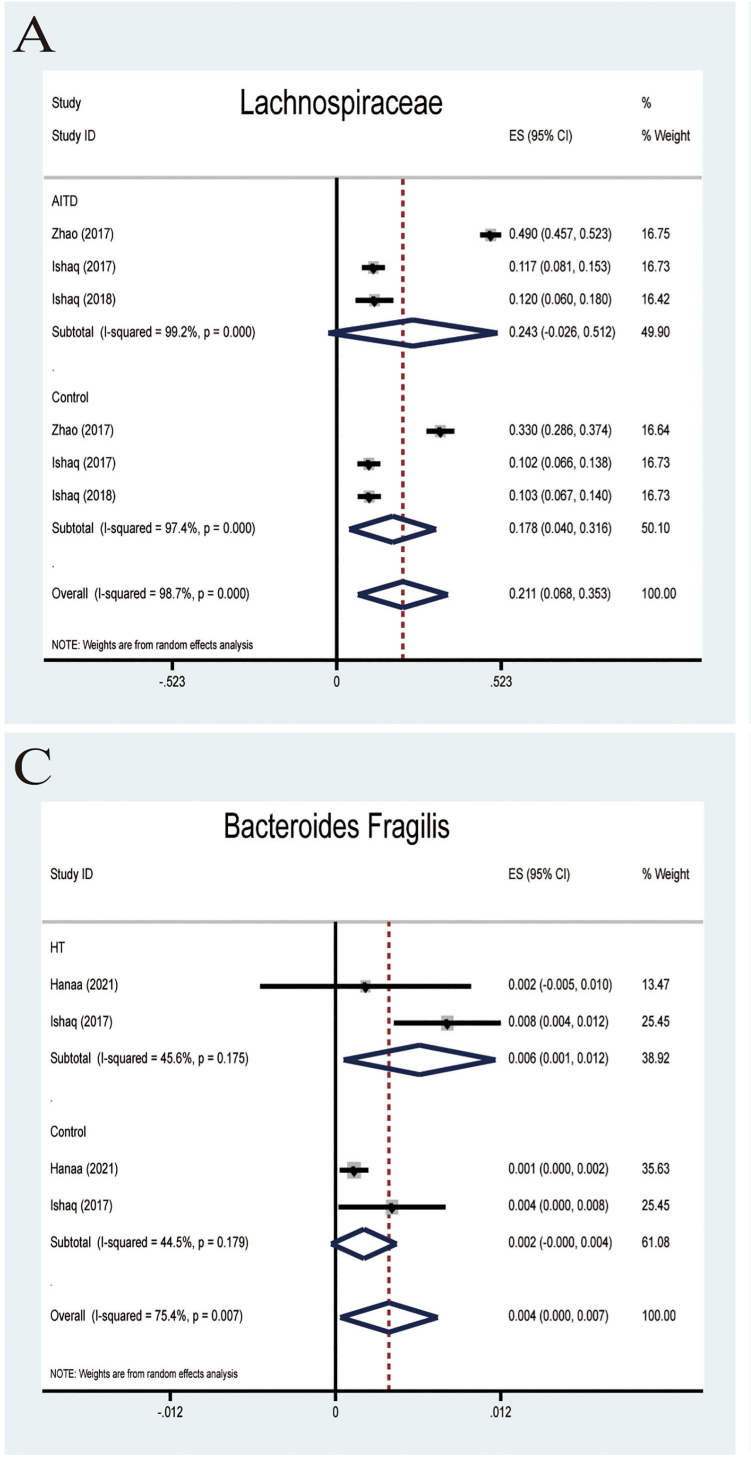

B

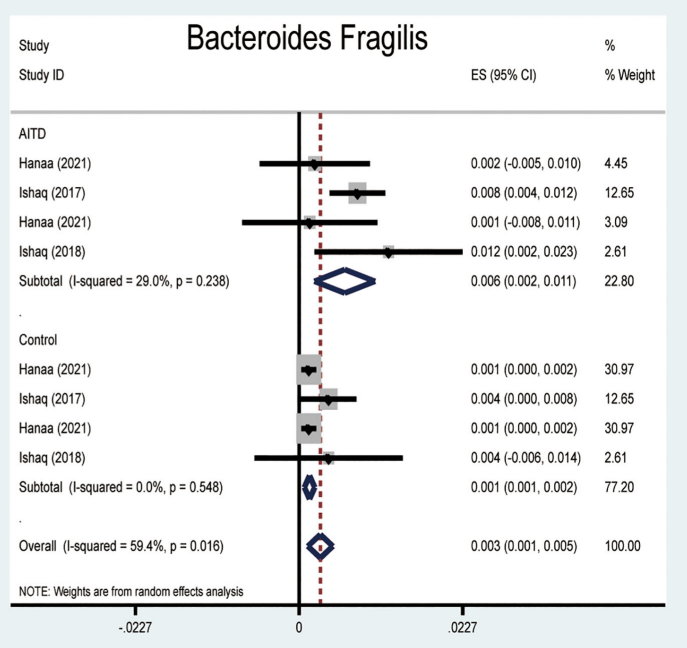

$\mathrm{D}$

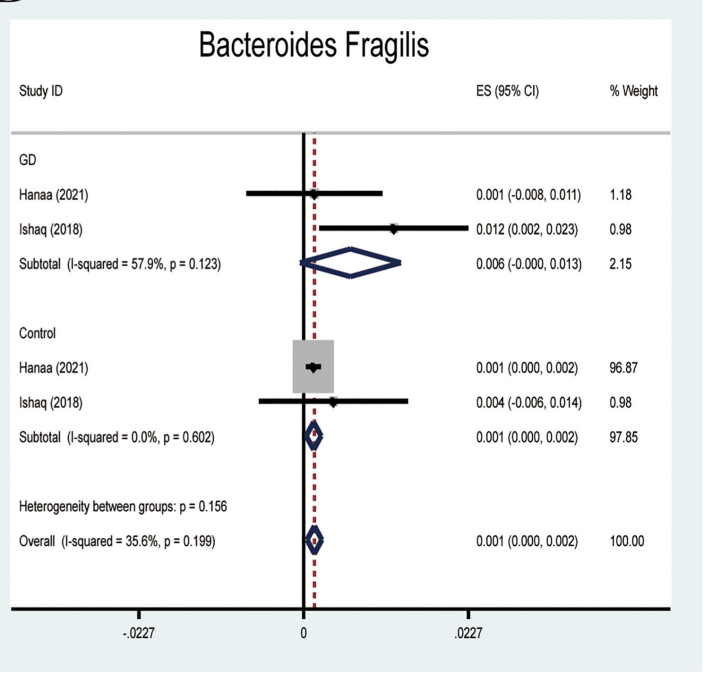

FIGURE 5 | Forest plot of the percentages of Lachnospiraceae and Bacteroides Fragilis in autoimmune thyroid disease (AITD). (A) A random-effects model was used to assess the percentages of Lachnospiraceae, contributing to higher between-study heterogeneity (I2>50\%). (B) A fixed-effects model was used to assess Bacteroides Fragilis, comparing patients with AITD to healthy controls. (C, D) Random-effects models were used to assess the percentages of Bacteroides Fragilis comparing people with Graves' disease (GD) or Hashimoto Thyroiditis (HT) to healthy controls. 

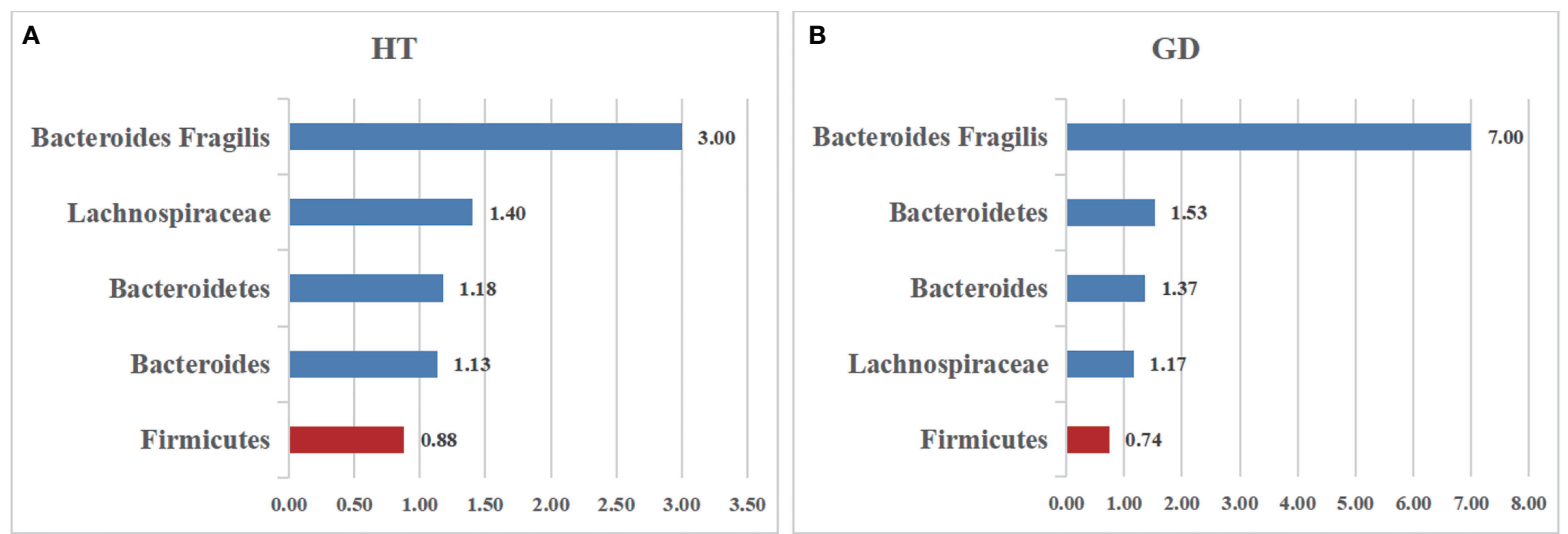

FIGURE 6 | The relative abundance of the included bacteria in our meta-analysis. (A) The ratio of the gut microbiota percentages in patients with HT. (B) The ratio of the bacteria percentages of patients with GD. A value greater than 1 shows a higher abundance of microbiota (Bacteroides Fragilis, Lachnospiraceae, Bacteroidetes, Bacteroides), whereas a value less than 1 indicates lower abundance compared to the healthy controls. (Firmicutes).

and underlying mechanism linking AITD and microbiota, expecting to discover a novel target for early diagnosis or reversal of the host's disordered immune system through fecal microbiota transplantation. Owing to the development of 16SrRNA sequencing technologies, significant alterations of the abundance and composition of gut microbiota have been found between AITD and healthy controls, suggesting that the gutthyroid axis may play a pivotal role in the development and progress of AITD (11).

Though HT and GD are considered the most common two forms of AITD, the pathogenesis and hallmark of HT and GD are quite different. The hallmark of HT is the high rate of TPO$\mathrm{Ab}$, and TG-Ab, while high TR-Ab level was detected in patients with GD (39). Besides, the alteration of certain gut microbiota compositions may be different between HT and GD. For example, the species community richness index Chaol was significantly elevated in samples from patients with HT while reduced in patients with GD. Species richness is an important feature of ecological composition and community structure.
The opposite indicates that HT patients have greater gut microbiota richness, which may be related to the bacterial overgrowth in the intestinal tract. In contrast, the GD patients may have less community abundance.

AITD has been linked to gut microbiota dysbiosis by different mechanisms, such as bacterial overgrowth, overactivation of the inflammasome, increased intestinal permeability, alteration of microbiota metabolites, and immune homeostasis (40). However, the mechanisms of the complex gut-thyroid axis have not been fully elucidated. Firmicutes and Bacteroidetes are the main dominant microbiota at the phyla level. Traditionally, the ratio of Firmicutes and Bacteroidetes has been implicated in the predisposition of disease conditions. In our meta-analysis, the rate of Firmicutes and Bacteroidetes ratio of patients with AITD showed a lower level than their healthy counterparts. Besides, AITD patients showed a notable alteration in gut microbiota composition as compared to the controls. A random-effect model indicates that people with AITD showed a significantly increased relative abundance of pathogenic

TABLE 3 | Potential correlation relationship between thyroid-related antibodies and gut microbiota.

\begin{tabular}{|c|c|c|c|c|c|}
\hline \multirow[t]{2}{*}{ Study } & \multirow[t]{2}{*}{ Family/Genus level } & \multicolumn{2}{|c|}{ TPOAb } & \multicolumn{2}{|c|}{ TRAb } \\
\hline & & $\mathbf{r}$ & $\mathbf{P}$ & $\mathbf{r}$ & $\mathbf{P}$ \\
\hline \multirow[t]{4}{*}{ Jiang,2020 } & Blautia & 0.365 & 0.014 & - & - \\
\hline & Bacteroides & -0.342 & 0.021 & - & - \\
\hline & Lactobacillus & 0.156 & $>0.05$ & 0.092 & $>0.05$ \\
\hline & Dorea & -0.341 & 0.022 & - & - \\
\hline \multirow[t]{4}{*}{ Cornejo,2020 } & Alistipes & 0.432 & 0.019 & - & - \\
\hline & Faecalibacterium & -0.453 & 0.014 & - & - \\
\hline & Ruminococcaceae & 0.408 & 0.028 & - & - \\
\hline & Enterobacteraceae & 0.416 & 0.025 & - & - \\
\hline \multirow[t]{4}{*}{ Chen,2020 } & Synergistetes & -0.711 & 0.001 & -0.702 & 0.000 \\
\hline & Lactobacillus & 0.607 & 0.006 & 0.489 & 0.024 \\
\hline & Corprococus & -0.499 & 0.030 & -0.366 & 0.103 \\
\hline & Phascolarctobacterium & -0.336 & 0.160 & -0.544 & 0.011 \\
\hline
\end{tabular}

TPO-Ab, thyroid peroxidase antibody; TR-Ab, thyrotropin receptor antibodies. 
bacteria and a decreased proportion of beneficial bacteria such as Lactobacillus and Bifidobacterium. Lactobacillus and Bifidobacterium could be used as a probiotic to modulate the immune response and have no adverse effect on developing an experimental autoimmune thyroiditis mice model (41). In addition, Lactobacillus has been proved to protect TH17 cells and support barrier integrity by secreting IL-22 and IL-17. The Th17/Treg imbalance may cause inflammatory disorders, indicating that Lactobacillus participates in immune system balance. Overall, Bifidobacterium and Lactobacillus showed antiinflammatory effects and shield our body from pathogen. What's more the Increased $B$. fragilis species may account for the upregulation of IL-18, IL-1 $\beta$, and caspase-1, promoting the inflammatory response. Besides, $B$. fragilis can active the expression of NLRP3, which have been found overexpression in HT patients. However, the interaction between gut microbiota and inflammasome was still unclear. Another reasonable hypothesis of the role that the microbiota plays in the progress of AITD is molecular mimicry. The Antigenic properties of proteins of certain intestinal bacteria may bind $\mathrm{TPO}-\mathrm{Ab}$ and TG-Ab, which are the main AITD's clinical diagnostic parameters (42).

Accumulating evidence suggests that the gut microbiota can regulate the local intestinal immune system and moderate the immune system outside the gastrointestinal system by metabolites secretion. The immunological system resulting in the development of AITD is potentially correlated with microbial metabolism. SCFAs, mainly butyrate, acetate, and propionate, are primarily produced by Bacteroidetes, Bifidobacterium, Faecalibacterium, and Enterobacteria, which are rich sources of energy for the host (43). Butyrate is one of the most important metabolites produced by butyrate-producing bacteria such as Firmicutes, enhancing the intestinal barrier and mucosal immunity. Thus, the decreased phylum Firmicutes in the AITD group may account for the increased intestinal permeability. Studies found a notable increase in the expression of inflammasome components, including NLRP1, NLRP3,NLRC4, and AIM2 in HT patients, which can be activated by intestinal microbiota through PAMPs (pathogen-associated molecular pattern molecules (PAMPs) or DAMPs (damageassociated molecular pattern molecules (DAMPs) patterns. Notably, the IL-1 $\beta$, the subsequent effector molecules downstream of inflammasomes, can modulate the microbiota by regulating the production of antimicrobial peptides (AMPs) (15). In addition, trimethylamine N-oxide (TMAO), a gut microbiota-dependent product, has been found to participate in the progression of vascular calcification and endothelial dysfunction by enhancing the activation and formation of the NLRP3 inflammasome, ASC, IL-1 $\beta$, and caspase-1 (44). A study including a 1621 Czech population also supports the connection between Helicobacter pylori and HT. H. pylori, a gram-negative bacterium of the human microbiota, predominantly promotes the NLRP3 inflammasome and caspase- 1 activation as well as IL-1 $\beta$ secretion via TLR4,MyD88, and NF-kB $(45,46)$. Moreover, certain microbiota may increase the permeability, allowing the toxins, antigens, or SCFA to pass into the blood circulation by activating the inflammatory system.
Furthermore, several correlation studies were performed to establish a relationship between thyroid autoimmunity parameters and specific bacteria based on Spearman correlation distance analysis. Spearman correlation analysis is one of the most common analysis to reveal the correlations between altered microbiota and various clinical parameters. In our study, the association between gut microbiota and AITD has already been explored, including the percent of certain microbiota increased or decreased compared to healthy controls. However, the altered abundance of gut microbiota may not indicate a direct association between gut microbiota and AITD. To bridge this gap, the correlation between specific microbiota and two clinical parameters (TPOAb and TRAb) of thyroid may provide guidance for the future study or diagnosis of AITD. We found that some intestinal microbiota, such as Ruminococcaceae, Blautia, and Alistipes, were significantly positively correlated with TPOAb. In contrast, other bacteria such as Bacteroides and Synergistetes were negatively correlated with TPO-Ab or TR-Ab. Furthermore, the gut microbiota is correlated with cell functions such as membrane transport, genetic information, or other cellular processes (30). Notably, the increased level of antibodies and thyroid hormone in AITD patients might affect and change the composition and amount of intestinal microbiota $(47,48)$. Therefore, we hypothesized that the AITD might be associated with the alterations of the gut microbiota.

\section{Limitation of the Study}

Several limitations of this meta-analysis should be considered. First, most studies did not consider the effect of dietary habits. The consumption of various food or fiber can influence the composition of the commensal microbiota and its metabolites, SCFA (47). Second, many of the included observational studies did not evaluate thyroid hormone influences and thyroid autoantibodies on gut microbiota composition. Third, seasonal variations influence gut microbiota composition. For example, the relative abundance of certain microbiota may be affected by the time of stool sample collection; however, only one study mentioned that stool samples were collected in Autumn. Fourth, a series of metabolism changes of AITD could also altered the composition of gut microbiota, thus, the results can only reflect an association between gut microbiota and autoimmune thyroid disease but not a causation relationship.

\section{CONCLUSION}

This meta-analysis shows the interactions between the gut microbiota and the pathogenesis of AITD. Furthermore, certain beneficial intestinal bacteria decrease, such as Bifidobacterium and Lactobacillus, may be associated with the development of AITD to some extent. However, the way how microbiome affects the progress of AITD is still controversial, and the research data is limited. Therefore, a further multicenter approach is needed to clarify the underlying pathogenesis and progress of intestinal microbiota dysbiosis in AITD. 


\section{DATA AVAILABILITY STATEMENT}

The original contributions presented in the study are included in the article/supplementary material. Further inquiries can be directed to the corresponding author.

\section{AUTHOR CONTRIBUTIONS}

BG and ZS conceived the study. CW, FM, HW, and YY participated in the statistical analysis. BG and BS drafted the article. All authors read and approved the final version of the manuscript.

\section{REFERENCES}

1. Lee HJ, Li CW, Hammerstad SS, Stefan M, Tomer Y. Immunogenetics of Autoimmune Thyroid Diseases: A Comprehensive Review. J Autoimmun (2015) 64:82-90. doi: 10.1016/j.jaut.2015.07.009

2. Banga JP, Schott M. Autoimmune Thyroid Diseases. Horm Metab Res = Horm Stoffwechselforschung = Horm Metabol (2018) 50(12):837-9. doi: 10.1055/a-0799-5068

3. Prummel MF, Strieder T, Wiersinga WM. The Environment and Autoimmune Thyroid Diseases. Eur J Endocrinol (2004) 150(5):605-18. doi: 10.1530/eje.0.1500605

4. Benvenga S, Elia G, Ragusa F, Paparo SR, Sturniolo MM, Ferrari SM, et al. Endocrine Disruptors and Thyroid Autoimmunity. Best Pract Res Clin Endocrinol Metab (2020) 34(1):101377. doi: 10.1016/j.beem.2020.101377

5. Lahner E, Conti L, Cicone F, Capriello S, Cazzato M, Centanni M, et al. Thyro-Entero-Gastric Autoimmunity: Pathophysiology and Implications for Patient Management. Best Pract Res Clin Endocrinol Metab (2020) 34 (1):101373. doi: 10.1016/j.beem.2019.101373

6. Cellini M, Santaguida MG, Virili C, Capriello S, Brusca N, Gargano L, et al. Hashimoto's Thyroiditis and Autoimmune Gastritis. Front Endocrinol (2017) 8:92. doi: $10.3389 /$ fendo.2017.00092

7. Kohling HL, Plummer SF, Marchesi JR, Davidge KS, Ludgate M. The Microbiota and Autoimmunity: Their Role in Thyroid Autoimmune Diseases. Clin Immunol (Orlando Fla) (2017) 183:63-74. doi: 10.1016/ j.clim.2017.07.001

8. Han H, Li Y, Fang J, Liu G, Yin J, Li T, et al. Gut Microbiota and Type 1 Diabetes. Int J Mol Sci (2018) 19(4):995. doi: 10.3390/ijms19040995

9. Mu Q, Zhang H, Liao X, Lin K, Liu H, Edwards MR, et al. Control of Lupus Nephritis by Changes of Gut Microbiota. Microbiome (2017) 5(1):73. doi: 10.1186/s40168-017-0300-8

10. Zhang X, Zhang D, Jia H, Feng Q, Wang D, Liang D, et al. The Oral and Gut Microbiomes Are Perturbed in Rheumatoid Arthritis and Partly Normalized After Treatment. Nat Med (2015) 21(8):895-905. doi: 10.1038/nm.3914

11. Knezevic J, Starchl C, Tmava Berisha A, Amrein K. Thyroid-Gut-Axis: How Does the Microbiota Influence Thyroid Function? Nutrients (2020) 12 (6):1769. doi: 10.3390/nu12061769

12. Cayres LCF, de Salis LVV, Rodrigues GSP, Lengert AVH, Biondi APC, Sargentini LDB, et al. Detection of Alterations in the Gut Microbiota and Intestinal Permeability in Patients With Hashimoto Thyroiditis. Front Immunol (2021) 12:579140. doi: 10.3389/fimmu.2021.579140

13. Tomasello G, Tralongo P, Amoroso F, Damiani P, Sinagra E, Noto M, et al. Dysmicrobism Inflammatory Bowel Disease and Thyroiditis: Analysis of the Literature. J Biol Regul Homeost Agents (2015) 29(2):265-72.

14. Guo Q, Wu Y, Hou Y, Liu Y, Liu T, Zhang H, et al. Cytokine Secretion and Pyroptosis of Thyroid Follicular Cells Mediated by Enhanced NLRP3, NLRP1, NLRC4, and AIM2 Inflammasomes Are Associated With Autoimmune Thyroiditis. Front Immunol (2018) 9:1197. doi: 10.3389/fimmu.2018.01197

15. Yao X, Zhang C, Xing Y, Xue G, Zhang Q, Pan F, et al. Remodelling of the Gut Microbiota by Hyperactive NLRP3 Induces Regulatory T Cells to Maintain Homeostasis. Nat Commun (2017) 8(1):1896. doi: 10.1038/s41467-017-01917-2

16. Chen GY. Regulation of the Gut Microbiome by Inflammasomes. Free Radical Biol Med (2017) 105:35-40. doi: 10.1016/j.freeradbiomed.2016.11.011

\section{FUNDING}

This study was supported by the Department of Education in Liaoning Province, China (LJKZ0742).

\section{ACKNOWLEDGMENTS}

We greatly thank David Moher and his international adversary group in the PROSPERO online platform, who have patiently checked my submitted manuscript. (The PROSPERO registration number is: CRD42021251557).

17. Vierbuchen T, Bang C, Rosigkeit H, Schmitz RA, Heine H. The HumanAssociated Archaeon Methanosphaera Stadtmanae Is Recognized Through Its RNA and Induces TLR8-Dependent NLRP3 Inflammasome Activation. Front Immunol (2017) 8:1535. doi: 10.3389/fimmu.2017.01535

18. Yang W, Yu T, Huang X, Bilotta AJ, Xu L, Lu Y, et al. Intestinal MicrobiotaDerived Short-Chain Fatty Acids Regulation of Immune Cell IL-22 Production and Gut Immunity. Nat Commun (2020) 11(1):4457. doi: 10.1038/s41467-020-18262-6

19. Ratajczak W, Ry A, Mizerski A, Walczakiewicz K, Sipak O, Laszczynska M. Immunomodulatory Potential of Gut Microbiome-Derived Short-Chain Fatty Acids (SCFAs). Acta Biochim Pol (2019) 66(1):1-12. doi: 10.18388/ abp.2018_2648

20. Pan X, Fang X, Wang F, Li H, Niu W, Liang W, et al. Butyrate Ameliorates Caerulein-Induced Acute Pancreatitis and Associated Intestinal Injury by Tissue-Specific Mechanisms. Br J Pharmacol (2019) 176(23):4446-61. doi: $10.1111 / \mathrm{bph} .14806$

21. Zhao F, Feng J, Li J, Zhao L, Liu Y, Chen H, et al. Alterations of the Gut Microbiota in Hashimoto's Thyroiditis Patients. Thyroid Off J Am Thyroid Assoc (2018) 28(2):175-86. doi: 10.1089/thy.2017.0395

22. Shi TT, Xin Z, Hua L, Zhao RX, Yang YL, Wang H, et al. Alterations in the Intestinal Microbiota of Patients With Severe and Active Graves' Orbitopathy: A Cross-Sectional Study. J Endocrinol Invest (2019) 42(8):967-78. doi: 10.1007/s40618-019-1010-9

23. Liu S, An Y, Cao B, Sun R, Ke J, Zhao D. The Composition of Gut Microbiota in Patients Bearing Hashimoto's Thyroiditis With Euthyroidism and Hypothyroidism. Int J Endocrinol (2020) 2020:5036959. doi: 10.1155/2020/5036959

24. Kim BR, Shin J, Guevarra R, Lee JH, Kim DW, Seol KH, et al. Deciphering Diversity Indices for a Better Understanding of Microbial Communities. J Microbiol Biotechnol (2017) 27(12):2089-93. doi: 10.4014/jmb.1709.09027

25. Ishaq HM, Mohammad IS, Guo H, Shahzad M, Hou YJ, Ma C, et al. Molecular Estimation of Alteration in Intestinal Microbial Composition in Hashimoto's Thyroiditis Patients. Biomed Pharmacother = Biomed Pharmacother (2017) 95:865-74. doi: 10.1016/j.biopha.2017.08.101

26. Ishaq HM, Mohammad IS, Shahzad M, Ma C, Raza MA, Wu X, et al. Molecular Alteration Analysis of Human Gut Microbial Composition in Graves' Disease Patients. Int J Biol Sci (2018) 14(11):1558-70. doi: 10.7150/ijbs.24151

27. Su X, Yin X, Liu Y, Yan X, Zhang S, Wang X, et al. Gut Dysbiosis Contributes to the Imbalance of Treg and Th17 Cells in Graves' Disease Patients by Propionic Acid. J Clin Endocrinol Metab (2020) 105(11):dgaa511. doi: 10.1210/clinem/dgaa511

28. Cornejo-Pareja I, Ruiz-Limon P, Gomez-Perez AM, Molina-Vega M, Moreno-Indias I, Tinahones FJ. Differential Microbial Pattern Description in Subjects With Autoimmune-Based Thyroid Diseases: A Pilot Study. J Personalized Med (2020) 10(4):192. doi: 10.3390/jpm10040192

29. Chen J, Wang W, Guo Z, Huang S, Lei H, Zang P, et al. Associations Between Gut Microbiota and Thyroidal Function Status in Chinese Patients With Graves' Disease. J Endocrinol Invest (2021) 44(9):1913-26. doi: 10.1007/ s40618-021-01507-6

30. Jiang W, Yu X, Kosik RO, Song Y, Qiao T, Tong J, et al. Gut Microbiota May Play a Significant Role in the Pathogenesis of Graves' Disease. Thyroid Off J Am Thyroid Assoc (2021) 31(5):810-20. doi: 10.1089/thy.2020.0193

31. Yang M, Sun B, Li J, Yang B, Xu J, Zhou X, et al. Alteration of the Intestinal Flora may Participate in the Development of Graves' Disease: A Study 
Conducted Among the Han Population in Southwest China. Endocr Connect (2019) 8(7):822-8. doi: 10.1530/ec-19-0001

32. El-Zawawy HT, Ahmed SM, El-Attar EA, Ahmed AA, Roshdy YS, Header DA. Study of Gut Microbiome in Egyptian Patients With Autoimmune Thyroid Diseases. Int J Clin Pract (2021) 75(5):e14038. doi: 10.1111/ ijcp. 14038

33. Zhou L, Li X, Ahmed A, Wu D, Liu L, Qiu J, et al. Gut Microbe Analysis Between Hyperthyroid and Healthy Individuals. Curr Microbiol (2014) 69 (5):675-80. doi: 10.1007/s00284-014-0640-6

34. Jandhyala SM, Talukdar R, Subramanyam C, Vuyyuru H, Sasikala M, Nageshwar Reddy D. Role of the Normal Gut Microbiota. World J Gastroenterol (2015) 21(29):8787-803. doi: 10.3748/wjg.v21.i29.8787

35. Abdelhamid AG, El-Masry SS, El-Dougdoug NK. Probiotic Lactobacillus and Bifidobacterium Strains Possess Safety Characteristics, Antiviral Activities and Host Adherence Factors Revealed by Genome Mining. EPMA J (2019) 10 (4):337-50. doi: 10.1007/s13167-019-00184-z

36. Nitzan O, Elias M, Peretz A, Saliba W. Role of Antibiotics for Treatment of Inflammatory Bowel Disease. World J Gastroenterol (2016) 22(3):1078-87. doi: 10.3748/wjg.v22.i3.1078

37. Vacca M, Celano G, Calabrese FM, Portincasa P, Gobbetti M, De Angelis M. The Controversial Role of Human Gut Lachnospiraceae. Microorganisms (2020) 8(4):573. doi: 10.3390/microorganisms 8040573

38. Yang PC, Li XJ, Yang YH, Qian W, Li SY, Yan CH, et al. The Influence of Bifidobacterium Bifidum and Bacteroides Fragilis on Enteric Glial CellDerived Neurotrophic Factors and Inflammasome. Inflammation (2020) 43 (6):2166-77. doi: 10.1007/s10753-020-01284-z

39. Dong YH, Fu DG. Autoimmune Thyroid Disease: Mechanism, Genetics and Current Knowledge. Eur Rev Med Pharmacol Sci (2014) 18(23):3611-8.

40. Manukyan GP, Ghazaryan KA, Ktsoyan ZA, Khachatryan ZA, Arakelova KA, Kelly D, et al. Elevated Systemic Antibodies Towards Commensal Gut Microbiota in Autoinflammatory Condition. PloS One (2008) 3(9):e3172. doi: 10.1371/journal.pone.0003172

41. Zhou JS, Gill HS. Immunostimulatory Probiotic Lactobacillus Rhamnosus HN001 and Bifidobacterium Lactis HN019 do Not Induce Pathological Inflammation in Mouse Model of Experimental Autoimmune Thyroiditis. Int J Food Microbiol (2005) 103(1):97-104. doi: 10.1016/ j.ijfoodmicro.2004.11.031

42. Kiseleva EP, Mikhailopulo KI, Sviridov OV, Novik GI, Knirel YA, Szwajcer Dey E. The Role of Components of Bifidobacterium and Lactobacillus in Pathogenesis and Serologic Diagnosis of Autoimmune Thyroid Diseases. Benef Microbes (2011) 2(2):139-54. doi: 10.3920/bm2010.0011
43. Fenneman AC, Rampanelli E, Yin YS, Ames J, Blaser MJ, Fliers E, et al. Gut Microbiota and Metabolites in the Pathogenesis of Endocrine Disease. Biochem Soc Trans (2020) 48(3):915-31. doi: 10.1042/bst20190686

44. Zhang X, Li Y, Yang P, Liu X, Lu L, Chen Y, et al. Trimethylamine-N-Oxide Promotes Vascular Calcification Through Activation of NLRP3 (NucleotideBinding Domain, Leucine-Rich-Containing Family, Pyrin Domain-Containing3) Inflammasome and NF-kB (Nuclear Factor Kb) Signals. Arterioscler Thromb Vasc Biol (2020) 40(3):751-65. doi: 10.1161/atvbaha.119.313414

45. Koch KN, Muller A. Helicobacter Pylori Activates the TLR2/NLRP3/caspase1/IL-18 Axis to Induce Regulatory T-Cells, Establish Persistent Infection and Promote Tolerance to Allergens. Gut Microbes (2015) 6(6):382-7. doi: 10.1080/19490976.2015.1105427

46. Seo SU, Kamada N, Munoz-Planillo R, Kim YG, Kim D, Koizumi Y, et al. Distinct Commensals Induce Interleukin-1 $\beta$ via NLRP3 Inflammasome in Inflammatory Monocytes to Promote Intestinal Inflammation in Response to Injury. Immunity (2015) 42(4):744-55. doi: 10.1016/j.immuni.2015.03.004

47. Ihnatowicz P, Drywien M, Wator P, Wojsiat J. The Importance of Nutritional Factors and Dietary Management of Hashimoto's Thyroiditis. Ann Agric Environ Med AAEM (2020) 27(2):184-93. doi: 10.26444/aaem/112331

48. Virili C, Fallahi P, Antonelli A, Benvenga S, Centanni M. Gut Microbiota and Hashimoto's Thyroiditis. Rev Endocr Metab Disord (2018) 19(4):293-300. doi: $10.1007 /$ s11154-018-9467-y

Conflict of Interest: The authors declare that the research was conducted in the absence of any commercial or financial relationships that could be construed as a potential conflict of interest.

Publisher's Note: All claims expressed in this article are solely those of the authors and do not necessarily represent those of their affiliated organizations, or those of the publisher, the editors and the reviewers. Any product that may be evaluated in this article, or claim that may be made by its manufacturer, is not guaranteed or endorsed by the publisher.

Copyright $\odot 2021$ Gong, Wang, Meng, Wang, Song, Yang and Shan. This is an openaccess article distributed under the terms of the Creative Commons Attribution License (CC BY). The use, distribution or reproduction in other forums is permitted, provided the original author(s) and the copyright owner(s) are credited and that the original publication in this journal is cited, in accordance with accepted academic practice. No use, distribution or reproduction is permitted which does not comply with these terms. 